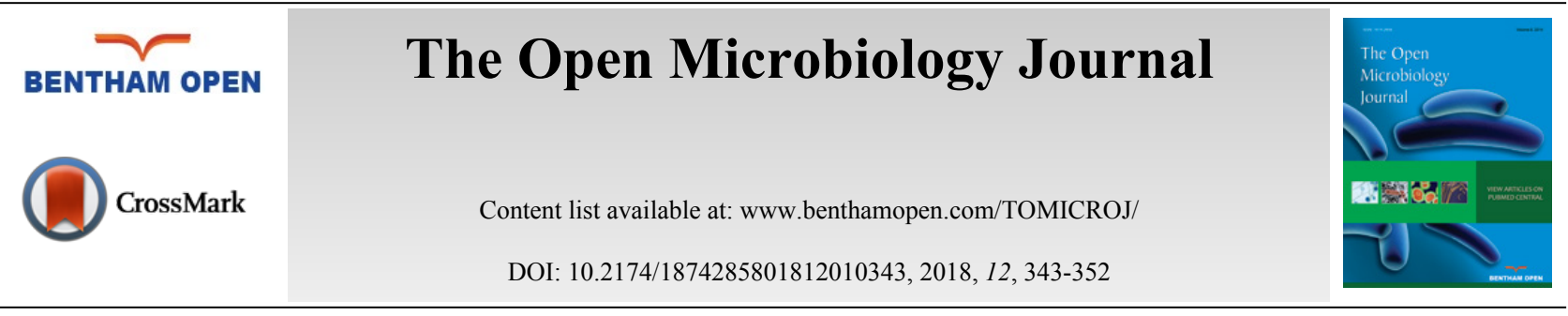

RESEARCH ARTICLE

\title{
First Detection of Chlamydia trachomatis 'Swedish' Variant (nvCT) in a Russian Couple with Infertility
}

Valentina Feodorova ${ }^{1, *}$, Edgar Sultanakhmedov², Yury Saltykov ${ }^{1}$, Sergey Zaitsev ${ }^{1}$, Sergey Utz ${ }^{2}$, Michael Corbel $^{3}$, Charlotte Gaydos ${ }^{4}$, Thomas Quinn ${ }^{4,5}$ and Vladimir Motin ${ }^{6, *}$

${ }^{I}$ Laboratory for Molecular Biology and NanoBiotechnology, Federal Research Center for Virology and Microbiology, Branch in Saratov, Ap. 6, the $53^{\text {rd }}$ Strelkovoi Divisii Street, Saratov, 410028, Russia

${ }^{2}$ Department for Skin Diseases, Saratov State Medical University, 22, Proviantskaya Street, Saratov, 410028, Russia

${ }^{3}$ Department of Bacteriology, The National Institute for Biological Standards and Control (NIBSC), Potters Bar, EN6 $3 Q G, U K$

${ }^{4}$ Division of Infectious Diseases, Johns Hopkins University School of Medicine, 855 N. Wolfe Street, Rangos Bldg, Suite 530, Baltimore, MD21205, USA

${ }^{5}$ Division of Intramural Research, National Institute of Allergy and Infectious Diseases, Baltimore, MD, 21205, USA

${ }^{6}$ Department of Pathology \& Immunology, University of Texas Medical Branch, 301 University Boulevard, Galveston, TX 77555-0609, USA

\section{Abstract:}

\section{Background:}

Chronic asymptomatic chlamydial genital infection caused by the wild-type of Chlamydia trachomatis (wtCT) is the most common bacterial infection causing human infertility. The novel 'Swedish' variant of C.trachomatis (nvCT) which contains a 377 bp deletion in a region that is specifically targeted in some nucleic acid amplification tests may impede diagnosis.

\section{Objective:}

The study aimed to investigate whether nvCT may be a possible cause of infertility in a couple undergoing in vitro fertilization (IVF).

\section{Method:}

Clinical specimens from both genital (urethra and cervix) and extra-genital sites (pharynx, conjunctive, blood) of a couple who experienced multiple unsuccessful attempts at pregnancy by natural fertilization and IVF procedures were analyzed before and after antibiotic therapy. Both partners had neither somatic nor endocrinal abnormality nor any clinically apparent genital manifestations of Chlamydia or other STIs.

\section{Results:}

Before antibiotic therapy all the samples of the Female Partner (FP) contained DNA of only the nvCT. After antibiotic therapy, additionally, DNA of wtCT of genovars E and D was detected in specimens from her conjunctiva and oropharynx. All samples of the Male Partner (MP) revealed co-infection of nvCT and wtCT. Identical SNP within the variable region 4 (VD4) of the ompA gene confirmed the identity of the wtCT strains found in both partners. The FP had a positive anti-chlamydial IgG titer. The sperm

\footnotetext{
* Address correspondence to this author at the Laboratory for Molecular Biology and NanoBiotechnology, Federal Research Center for Virology and Microbiology, Branch in Saratov, Ap. 6, the $53^{\text {rd }}$ Strelkovoi Divisii Street, Saratov, 410028, Russia; Department of Pathology \& Immunology, University of Texas Medical Branch, 301 University Boulevard, Galveston, TX 77555-0609, USA; Tel: 9053850120; E-mail: feodorovav@mail.ru, vlmotin@utmb.edu
} 
characteristics of the MP, motility (immotile spermatozoa was $51.1 \%$ versus $21.6 \%$ ) and vitality (46\% versus $68 \%$ ) declined progressively, and the MP anti-chlamydial IgG titer was negative.

\section{Conclusion:}

Infertility in this couple may have been caused by chronic asymptomatic and persistent nvCT-associated infection that was complicated by re-infection later with wtCT. This study illustrates the importance of including detection methods for nvCT strains in the investigation of infertility cases.

Keywords: Chlamydia trachomatis, Novel Swedish strain, Co-infection, Extra-genital site, Infertility, Detection methods.

\section{INTRODUCTION}

Chlamydia trachomatis is currently one of the most prevalent sexually transmitted pathogens worldwide and a major cause of infertility affecting $10-15 \%$ of all couples worldwide [1 - 4]. The essential role of the wild type of C.trachomatis (wtCT) in human infertility is well-established $[2,4]$. A number of animal studies also present clear evidence that human strains of wtCT are capable of causing infertility $[5,6]$. This wtCT-associated infection can result in alteration of semen parameters, induction of apoptosis in spermatozoa, and decrease in the reproductive performance of fertile male mice [5]. The relevance of this observation was confirmed in recent studies where in-vitro co-incubation of human sperm with wtCT (serovar E) caused a significant decline in the number of live and motile spermatozoa, stimulation of an apoptosis-like response in spermatozoa and premature sperm death $[2,7,8]$. In the female, wtCT is a major cause of pelvic inflammatory disease, leading to chronic abdominal pain, ectopic pregnancy and tubal factor infertility [1, 4]. The wtCT genital chlamydial infections are frequently chronic and asymptomatic [2, 4]. If undiagnosed, wtCT can produce poor outcomes with respect to infertility problems including failure of both natural and assisted conception techniques, such as in vitro fertilization (IVF) [4, 9]. Unfortunately, the actual contribution of chlamydial infection caused by the novel 'Swedish' variant of C.trachomatis (nvCT) to couple infertility remains poorly investigated. This chlamydial variant, which carries a $377 \mathrm{bp}$ deletion within the cryptic plasmid that has caused false-negative responses in routine nucleic acid amplification tests (NAATs) $[10,11]$ is a novel challenge for laboratory diagnosis of chlamydial infection worldwide. At first, this type of strain was difficult to detect with commercial NAATs, since the deletion was located within the orfl of the plasmid targeted by diagnostics. For the past ten years, the nvCT strains have been intensively studied with respect to their phenotypic and biological characteristics. Based on whole genome sequencing, molecular epidemiology and in vitro research data, characteristics of the nvCT were found to be similar to those of wtCT $[2,12-15]$. Nevertheless, the nvCT strains exceeded the wild type strains in their ability to disseminate rapidly in the human population that was attributed to the problems of initial difficulties in detection of such variants [12]. However, little is known about the contribution of the nvCT to the development of chronic persistent chlamydial genital infection, infertility, clinical manifestations, extra-genital forms of the disease, and their ability to compete with the wtCT during mixed infection. Also, it is important to determine whether the nvCT can induce chlamydial infection as a sole etiologic factor, or if it requires a co-infection with the wtCT variant. This knowledge should improve routine laboratory diagnostics and treatment of genital infection caused by both wtCT and nvCT.

In this study, we report an investigation of chronic asymptomatic chlamydial genital infection involving extensive molecular testing in a sub-fertile couple that underwent multiple unsuccessful attempts at pregnancy by both natural fertilization and IVF procedures.

\section{MATERIALS AND METHODS}

\subsection{Brief Personal and Clinical Characteristics of Patients}

Female and male partners of 25 and 28 years old, respectively, comprised a heterosexual married couple from Saratov city. During the past six years, they had frequent sexual contacts to attempt pregnancy. Moreover, this couple had been engaged in receptive oral and genital, but not anal intercourse. Each individual had regular annual physical and laboratory examinations by a gynecologist or andrologist, an endocrinologist and a family physician. Both partners had neither somatic nor endocrinal abnormality nor symptoms, nor any clinically apparent genital manifestations of Chlamydia or other Sexually Transmitted Infections (STIs). Nevertheless, they demonstrated marked symptoms of bacterial conjunctivitis, such as unilateral hyperemia and mucopurulent discharge that were observed for the last 6 months. During the last three years, the patients underwent several unsuccessful attempts at IVF. The female partner disclaimed any sexual contacts outside legal marriage. However, the male partner reported frequent and irregular 
relationships with at least 3 to 5 female partners annually. Both partners denied any pernicious habits or addiction to alcohol, as well as animal or homosexual contacts. Both of them declared that they had never traveled to Sweden or other EU countries.

\subsection{Clinical Samples}

Clinical specimens were taken in October 2013, from both genital (urethra \& cervix) and extra-genital (oropharynx, conjunctive, blood) sites of each individual one year after a single course of antibiotic therapy. Clinical samples from similar sites of both partners were also obtained in October, 2012 prior to initiation of antibiotic therapy according to recommendations of the European STD Guidelines, and CDC STDs Treatment Guidelines [16, 17]. All procedures performed in studies involving human participants were in accordance with the ethical standards of the 1964 Helsinki declaration and its later amendments or comparable ethical standards. The research protocol was approved by the Human Bioethics Committee of the Saratov Scientific and Research Veterinary Institute No. IRB00008288 (http://ohrp.cit.nih.gov/search/IrbDtl.aspx), and both participants provided written informed consent.

\subsection{Semen Analysis}

Semen analysis was performed on the male partner according to the procedures outlined by the World Health Organization (WHO) [18], twice in October 2012, and three times in August 2014 by means of an automated sperm analyzer SQA-V (MES, Austria-Israel).

\subsection{Detection of C.trachomatis by Monoclonal Antibodies}

Direct Immunofluorescent Test (DIFT) with a mixture of chlamydia monoclonal antibodies (MAbs) to synthetic serogroup-specific MOMP-associated epitopes of 17 C.trachomatis serovars A-L (NiarMedic Plus, Moscow, Russia) [19] was used to test for chlamydial Elementary Bodies (EBs) in individual clinical samples.

\subsection{Detection of C.trachomatis Plasmid and Chromosomal DNA by PCR and Genotyping}

Total DNA was isolated from clinical samples including blood specimens using DNeasy Blood and Tissue Kit (Qiagen, Hilden, Germany). Each DNA sample was tested by: (i) End-point PCR with conventional and fluorescent product detection using commercial kits developed by the Central Research Institute of Epidemiology, Moscow, Russia (AmpiSens-Eph and AmpiSens-FL, respectively) to reveal the plasmid DNA of the typical C. trachomatis strains (wtCT) in the clinical material [20]; (ii) PCR with two pairs of primers, such as orf2_F/orf2_R and orf8_F/orf8_R (Table 1), designed by us to recognize genes orf2 and orf8 of C. trachomatis plasmid (PCR-orf2 and PCR-orf8, respectively).

Table 1. Primers used to amplify $C$. trachomatis DNA ${ }^{a}$.

\begin{tabular}{|l|l|l|}
\hline Primer & Sequence & Reference \\
\hline orf8_F & TCTTCTGCTTACAATGCTCTTGC & This study \\
\hline orf__R & CATCGACCTTGGTTTTTAAATCG & This study \\
\hline orf__F & AGCGAGTTACGAAGACAAAACCTC & This study \\
\hline orf__R & AGATTTGTTTCCAACAAGCTACCA & This study \\
\hline swCT_serE_F & TCCGGATAGTGAATTATAGAGACTATTTAATC & {$[1]$} \\
\hline swCT_serE_R & GGTGTTTGTACTAGAGGAATTACCTCTTC & This study \\
\hline Fw1 & TTCAATTTAGTTGGATTGTTTGG & {$[2]$} \\
\hline Fw2 & TCAACTTAGTTGGCTTATTCGG & {$[2]$} \\
\hline Fw3 & TCAATTTAGTGGGGTTATTCGG & {$[2]$} \\
\hline Rv1 & CACATTCCCAGAGAGCTGC & {$[2]$} \\
\hline Rv2 & CACATTCCCACAAAGCTGC & {$[2]$} \\
\hline Rv3 & CGGACTCCCACAAAGCTGC & {$[2]$} \\
\hline Rv4 & GCACTCCCACAAAGCTGC & {$[2]$} \\
\hline F11 & ACCACTTGGTGTGACGCTATCAG & {$[3]$} \\
\hline B11 & CGGAATTGTGCATTTACGTGAG & {$[3]$} \\
\hline MOPM-E_F1.seq & CGGTATTAGTATTTGCCGCTTTG & This study \\
\hline MOPM-E_F3.seq & CGACATATGCAGGATGCTGAG & This study \\
\hline MOPM-E_F5.seq & TACCATGAGTGGCAAGCAAGTT & This study \\
\hline
\end{tabular}


(Table $\square$ ) contd.....

\begin{tabular}{|l|l|l|}
\hline Primer & Sequence & Reference \\
\hline MOPM-E_R2. seq & GAATACATCAAAGCGATCCCAAA & This study \\
\hline MOPM-E_R4. seq & GATTGAGCGTATTGGAAAGAAGC & This study \\
\hline MOPM-E_R6. seq & TGCTCGAGACCATTTAACTCCA & This study \\
\hline
\end{tabular}

${ }^{a}$ Primers for this study were designed by the Primer 3 program (http://bioinfo.ut.ee/primer3-0.4.0/primer3/.

We used plasmid pBour of C. trachomatis strain E/Bour (GeneBank accession no. HE603212) to obtain sequences of both orf2 and orf8 genes. These primers amplified fragments of $150 \mathrm{bp}$ and $145 \mathrm{bp}$ for orf 2 and orf8, respectively. The reaction was done in a $30 \mu \mathrm{L}$ volume with $10 \mathrm{pmol}$ of each primer, and $1 \mu \mathrm{L}$ of DNA isolated from the clinical sample. The conditions of amplification were as follows: $94^{\circ} \mathrm{C}$ for $2 \mathrm{~min}, 15$ cycles of $94^{\circ} \mathrm{C}$ for $30 \mathrm{sec}, 61^{\circ} \mathrm{C}$ for 20 sec, and $72^{\circ} \mathrm{C}$ for $30 \mathrm{sec}$ followed by 45 cycles of $94^{\circ} \mathrm{C}$ for $30 \mathrm{sec}, 58^{\circ} \mathrm{C}$ for $20 \mathrm{sec}$, and $72^{\circ} \mathrm{C}$ for $45 \mathrm{sec}$ with the final extension at $72^{\circ} \mathrm{C}$ for $10 \mathrm{~min}$; (iii) PCR with the pair of primers developed by Catsburg et al. [21] to identify the presence of DNA of novel Swedish strains of $C$. trachomatis (nvCT) - PCR-nvCT, respectively. The forward primer swCT_serE_F was used in the original design. The reverse primer swCT_serE_R was modified by us in a single nucleotide to fit better the sequence of genovar E (Table 1). These primers amplified a product with a size of $474 \mathrm{bp}$ and $97 \mathrm{bp}$ in the presence of wtCT and nvCT templates, respectively. The DNA of genovar E of C. trachomatis strain E/Bour (\#VR-348BD, ATCC) was used as a control for each experiment. The reaction was done in $30 \mu \mathrm{L}$ with 10 pmol of each primer, and $1 \mu \mathrm{L}$ of DNA isolated from the clinical sample. The conditions of amplification were the following: $94^{\circ} \mathrm{C}$ for $2 \mathrm{~min}, 35 \mathrm{cycles}$ of $94^{\circ} \mathrm{C}$ for $30 \mathrm{sec}, 57^{\circ} \mathrm{C}$ for $30 \mathrm{sec}$, and $72^{\circ} \mathrm{C}$ for $45 \mathrm{sec}$ followed by the final extension at $72^{\circ} \mathrm{C}$ for $10 \mathrm{~min}$. Then, the fragments of amplification were purified from gel by using the QIAquick Gel Extraction Kit (Qiagen), and sequenced commercially by the EVROGEN Company (Moscow, Russia).

The individual C. trachomatis-positive DNA samples were genotyped by sequencing of the variable domain 2 (VD2) region of ompA as previously described by Quint et al. [22]. This system of genotyping included three forward and four reverse primers that in a multiplex format could amplify the VD2 region of all known C. trachmatis genovariants generating amplicons of 157 to $160 \mathrm{bp}$. The fragments of amplification were extracted from gel and sequenced. To determine the genovar, the sequence reads from the forward and reverse primers were searched in the GenBank for homology by the BLAST program.

The ompA gene was amplified with the use of primers F11 and B11, which produced the 1156 bp fragment containing all four variable regions of the gene [23]. To sequence the amplicons, additional primers complementary to the internal part of the ompA gene were designed (MOMP-E_F1.seq; MOMP-E_F3.seq; MOMP-E_F5.seq; MOMPE_R2.seq; MOMP-E_R4.seq; MOMP-E_R6.seq, Table 1, Fig. S1) allowing reading of the fragment sequence at least twice from the direct and reverse chains. The sequencing was done commercially by the EVROGEN Company. The consensus sequence of the $о т р A$ derived from the DNA of clinical sample was compared with the reference sequences of the strains of genovar E, such as E/Bour (HE601870), E/IU-TC0755ut (FJ261948), and E/H38 (AF265238), as well as strains of the genovar D, such as D/SotonD1 (HE601798), D/SotonD5 (HE601799) as reported by Harris et al. [13]. The nucleotide sequence alignment was done using the MultAlin (http://multalin.toulouse.inra.fr/multalin/multalin.html program).

All representative genovar sequences reported in this research were deposited in GenBank (accession numbers MF288583-MF288585).

\subsection{Detection of STIs DNA by PCR}

Each DNA sample was also tested by real-time multiplex commercial kit developed by the Vector-Best (Novosibirsk, Russia) to reveal DNA of Ureaplasma spp., Mycoplasma hominis, Mycoplasma genitalium, Trichomonas vaginalis, Neisseria gonorrhoeae, Candida albicans, Gardnerella vaginalis, cytomegalovirus, herpes simplex virus (HSV) types 1/2 and human papillomavirus (HPV) types 16 and 18 [20].

\subsection{Determination of Chlamydial IgG titres}

C.trachomatis immunoglobulin $\mathrm{G}$ (IgG) antibody titres (CAT) of the patients were tested with an ELISA kit ("VECTOR-BEST" Company, Novosibirsk-117, Russia) according to the manufacturer's instructions. Results were considered to be positive at ELISA titers $\geq 1: 32$. 


\section{RESULTS}

\subsection{Detection of nvCT in the Genital Samples of both Female and Male Patients}

The initial screening in October 2012 prior to the planned IVF procedure revealed the lack of C.trachomatis DNA in the genital specimens (cervix and urethra) of both partners using AmpliSens Chlamydia trachomatis-Eph and -FL kits (AmpiSens-Eph and AmpiSens-FL). Nevertheless, specific chlamydial antigens were identified in these clinical specimens by DIFT (data not shown).

Further we tested the samples for the presence of chlamydial DNA in PCR by using primers targeting different parts of the C. trachomatis cryptic plasmid (orf2 and orf8, PCR-orf2 and PCR-orf8), as well as primers swCT_serE_F/swCT_serE_R (Table 1) flanking the area of deletion identified in the orfl of the Swedish strains (nvCT, PCR-nvCT, respectively). Although amplification with the primers recognizing both orf 2 and orf 8 provided negative results, specific DNA of the Swedish nvCT variant bearing a cryptic plasmid with a 377-bp deletion in the orfl was detected in the samples from the genital sites tested. The exact position of the 377-bp specific deletion within the orf1 plasmid gene was confirmed by sequencing (data not shown). Additional examination of the female patient revealed scanty mucous vaginal discharge and moderate hyperemia in uterine and cervical mucosa in the absence of symptoms or any other clinical manifestations. After a single course of antibiotic therapy with doxycycline (Unidox Solutab $\left.{ }^{\circledR}\right) 100$ $\mathrm{mg}$ twice a day for seven days, and subsequent analysis of clinical specimens one year later (October 2013), her genital samples (urethra and cervix) were negative for wtCT DNA; however, the PCR analysis still revealed the presence of nvCT DNA in her cervix (Table 3). The urethral specimen of the male partner was also initially (October, 2012) positive for nvCT alone (Table 2). Later (October, 2013), in contrast to the results of the female patient testing, we detected chlamydial DNA with primers recognizing both wtCT and nvCT variants suggesting mixed infection. The presence of chlamydial EBs in clinical samples from genital sites of both partners was confirmed by DIFT (data not shown).

Table 2. Detection of Chlamydia from genital and extra genital sites of the female and male partners in clinical specimens obtained in 2012.

\begin{tabular}{|c|c|c|c|c|c|c|c|c|c|c|c|c|}
\hline \multirow{3}{*}{$\begin{array}{l}\text { Clinical } \\
\text { samples }\end{array}$} & \multicolumn{12}{|c|}{ Identification of $C$. trachomatis by PCR } \\
\hline & \multicolumn{6}{|c|}{ Female partner } & \multicolumn{6}{|c|}{ Male partner } \\
\hline & \begin{tabular}{|c|} 
AmpiSens- \\
Eph
\end{tabular} & \begin{tabular}{|c|} 
AmpiSens- \\
FL
\end{tabular} & $\underset{\text { a) }}{\text { PCR-nvCT }}$ & PCR-orf8 & PCR-orf2 & Genovar $^{\text {b) }}$ & $\begin{array}{c}\text { AmpiSens- } \\
\text { Eph }\end{array}$ & \begin{tabular}{|c|} 
AmpiSens- \\
FL
\end{tabular} & $\underset{\text { a) }}{\text { PCR-nvCT }}$ & PCR-orf8 & PCR-orf2 & Genovar $^{\text {b) }}$ \\
\hline Conjunctiva & - & - & + & + & - & E & - & - & + & + & - & E \\
\hline Oropharynx & - & - & + & + & - & $\mathrm{E}$ & - & - & + & - & - & $\mathrm{E}$ \\
\hline $\begin{array}{c}\text { Urethra \& } \\
\text { Cervix }\end{array}$ & - & - & + & - & - & $\mathrm{E}$ & - & - & + & + & - & $\mathrm{E}$ \\
\hline Blood & - & - & + & - & - & $\mathrm{E}$ & - & - & + & - & - & $\mathrm{E}$ \\
\hline
\end{tabular}

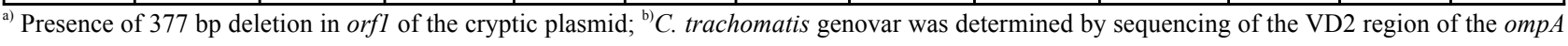
gene.

Table 3. Detection of Chlamydia from genital and extra genital sites of the female and male partners in clinical specimens obtained in 2013.

\begin{tabular}{|c|c|c|c|c|c|c|c|c|c|c|c|c|}
\hline \multirow{3}{*}{$\begin{array}{c}\text { Clinical } \\
\text { samples }\end{array}$} & \multicolumn{12}{|c|}{ Identification of $C$. trachomatis by PCR } \\
\hline & \multicolumn{6}{|c|}{ Female partner } & \multicolumn{6}{|c|}{ Male partner } \\
\hline & $\begin{array}{l}\text { Ampi } \\
\text { Sens- } \\
\text { Eph }\end{array}$ & $\begin{array}{l}\text { PCR- } \\
\text { nvCT }^{\text {a) }}\end{array}$ & $\begin{array}{l}\text { PCR- } \\
\text { orf2 }\end{array}$ & $\begin{array}{c}\text { PCR- } \\
\text { orf8 }\end{array}$ & $\begin{array}{l}\text { Geno- } \\
\text { var }^{\text {b) }}\end{array}$ & $\begin{array}{c}\text { SNP, } \\
\text { position }^{\text {c }}\end{array}$ & \begin{tabular}{|c|} 
Amp \\
i \\
Sens- \\
Eph \\
\end{tabular} & $\begin{array}{l}\text { PCR- } \\
\text { nvCT }^{\text {a }}\end{array}$ & $\begin{array}{l}\text { PCR- } \\
\text { orf } 2\end{array}$ & $\begin{array}{l}\text { PCR- } \\
\text { orf8 }\end{array}$ & $\begin{array}{l}\text { Geno- } \\
\text { var') }\end{array}$ & $\begin{array}{c}\text { SNP, } \\
\text { position } \\
\text {, }\end{array}$ \\
\hline \begin{tabular}{|c|} 
Conjunctiv \\
$\mathrm{a}$ \\
\end{tabular} & - & + & - & + & $\begin{array}{l}\mathrm{D} \\
\mathrm{E}\end{array}$ & $\begin{array}{c}991,1020 \\
\text { None }\end{array}$ & - & + & - & + & $\mathrm{E}$ & None \\
\hline Oropharynx & + & + & - & + & $\begin{array}{l}\mathrm{D} \\
\mathrm{E}\end{array}$ & $\begin{array}{c}971 \\
\text { None }\end{array}$ & - & + & + & - & $\begin{array}{l}\mathrm{D} \\
\mathrm{E}\end{array}$ & $\begin{array}{l}971 \\
\text { None }\end{array}$ \\
\hline \begin{tabular}{c|} 
Urethra \& \\
Cervix
\end{tabular} & - & + & - & - & $\mathrm{E}$ & None & + & + & - & + & $\mathrm{E}$ & None \\
\hline Blood & - & + & + & + & $\mathrm{E}$ & None & - & + & + & - & $\mathrm{E}$ & 997 \\
\hline
\end{tabular}

a) Presence of 377 bp deletion in orf1 of the cryptic plasmid; ${ }^{b)}$ C. trachomatis genovar was determined by sequencing of the VD2 region of the ompA gene; ${ }^{c}$ Single nucleotide polymorphism detected in the omp $A$ gene: the SNP position compared to the genovars E and D, strains E/Bour and $\mathrm{D} /$ SotonD5, respectively.

\subsection{Presence of Extra-genital C.trachomatis Infection}

During the first screening in October 2012, both AmpiSens-Eph and AmpiSens-FL kits failed to detect the wtCT 
DNA in extra-genital specimens of both partners. PCR-orf2 did not detect the presence of Chlamydia. Nevertheless, PCR-orf8 revealed Chlamydia in specimens derived from two out of three extra genital sites (conjunctiva and oropharynx). Moreover, PCR-nvCT produced positive signals with all three of their extra-genital samples, namely conjunctiva, oropharynx and blood (Table 2). Testing the specimens obtained one year later showed that both male and female patients were positive for Chlamydia in all extra-genital sites. AmpiSens-Eph and AmpiSens-FL were less efficient, although both PCR-orf2 and PCR-orf8 produced positive reactions in most specimens. PCR-nvCT detected Chlamydia in all samples. Overall, both wtCT and nvCT were detected indicating a mixed infection in extra-genital sites of this couple. Chlamydial EBs were found by DIFT in all clinical samples.

\subsection{Co-infection with nvCT and wtCT Genovars and Marker SNPs}

The ompA genotype was identified as genovar $\mathrm{E}$ in all clinical samples from genital sites and in each of the three extra-genital sites tested (Tables 2 and 3). Only novel 'Swedish' variant (nvCT) of C.trachomatis genovar E was detected in the patients at the first screening (Table 2). One year later, specific chlamydial DNA of genovar D was also found in extra-genital sites of both partners, namely in conjunctiva and oropharynx of the female partner and in oropharynx of her husband, indicating a mixed infection in these extra-genital sites (Table 3). When we determined the nucleotide sequence of the ompA gene of these genovar D variants, we identified SNPs that are not present in the current version of the GenBank for this genotype. The SNP at position 971 was matched in the samples obtained from the oropharynx of both partners, suggesting the identity of the strains that infected both patients. This SNP displayed a $\mathrm{C} \rightarrow \mathrm{A}$ substitution (compared with $\mathrm{D} /$ SotonD5) that resulted in an alanine-to-aspartic acid residue change within the VD4 region of the MOMP. Moreover, two additional SNPs were found in the conjunctiva of the female patient, those at positions 991 and 1020 (Table 3). The SNP at position 991 was also located within the VD4 region, and displayed an $\mathrm{A} \rightarrow \mathrm{G}$ substitution resulting in a threonine-to-alanine amino acid change. The SNP at position 1020 was mapped at the second amino acid after the end of the VD4 region. The $\mathrm{A} \rightarrow \mathrm{C}$ substitution did not change the amino acid residue. A single SNP for the genovar E was identified at position 997 in the blood sample of the male patient. This SNP displayed a $\mathrm{G} \rightarrow \mathrm{A}$ substitution (compared with E/Bour) that resulted in an alanine-to-threonine amino acid change within the VD4 region of the MOMP. The $о m p A$ sequence found in this clinical specimen was identical with that existing in the strains E/IU-TC0755ut and E/H38 found in the GenBank. Importantly, since AmpiSens-Eph and AmpiSens-FL recognized the typical strains of C.trachomatis (wtCT), such as E/Bour, PCR-nvCT detected the 'Swedish' variant with the 377-bp deletion in the orfl of the cryptic plasmid. The ompA sequence analysis revealed the strain with the SNP at position 997, and there were at least three different strains of the genovar E present in the clinical specimens of our two patients.

\subsection{Chlamydial IgG Titres in Sera of the Patients}

The female partner demonstrated a CAT IgG titre of 1:160 at both initial screen and on re-examination one year later. No CAT IgG was registered in the male partner during this study.

\subsection{Decreased Sperm Motility and Vitality in the Male Partner}

The characteristics of the sperm in samples obtained in 2014 in comparison with those taken in 2012: progressive were, $29.8 \%$ versus $45 \%$ (the lower reference limit is $32 \%$ ); non-progressive, $19.1 \%$ versus $23.4 \%$; immotile, $51.1 \%$ versus $21.6 \%$; vitality $46 \%$ versus $68 \%$ (the lower reference limit is $58 \%$ ); pH 7.9 versus 7.6 (normal range is $7.2-7.8$ ). Other major sperm parameters, such as total fluid volume, and spermatozoa concentration, were $4.5 \mathrm{ml}$ (the lower reference limit is $\geq 1,5 \mathrm{ml}$ ) and $76.5 \times 10^{6} / \mathrm{ml}$ (the lower reference limit is $15 \times 10^{6}$ spermatozoa per ml), respectively, were maintained at the normal level. Overall, the sperm quality of the male partner collected in 2014 had declined after two years of chlamydial infection.

\section{DISCUSSION}

In this study, we carefully investigated both asymptomatic partners in a couple with infertility and recurrent chlamydial genital infection. At the beginning of this investigation, the partners demonstrated neither clinical nor laboratory manifestations of infertility as indicated also by male initial semen analysis prior to their first visit to the IVF Center. Additionally, their genital specimens, including male first void urine, considered as the most acceptable sample for detection of C.trachomatis in men [2] was negative for C.trachomatis when tested by commercial Russian kits AmpliSens-Eph and-FL that could consistently detect plasmid DNA of the typical wtCT strains [20], as well as tests for other STIs. Nevertheless, the female partner was seropositive for chlamydial antigens. Therefore, both partners received adequate treatment (doxycycline, $100 \mathrm{mg}$ twice a day during 7 days) $[16,17]$ to prevent possible reinfection of 
this couple; however, not all the other multiple sexual partners of the male patient underwent antibiotic treatment.

Because multiple attempts at pregnancy conducted by the IVF procedure were unsuccessful, specimens from both female and male patients were investigated in our Reference Chlamydial laboratory, since a strong association between asymptomatic chlamydial genital infection in men and unexplained infertility has been suggested in literature [3]. In contrast to the new CDC Recommendations of 2014 for laboratory screening for Chlamydia in patients with and without symptoms of infection [24], the Russian standard laboratory protocol does not require testing of rectal samples from patients who are not engaged in anal intercourse. Therefore rectal specimens were omitted from this study, and cervical and urethral samples were analyzed. Additionally, we tested conjunctival, pharyngeal and blood specimens, since C.trachomatis antibodies can be detected in peripheral blood of patients with chronic asymptomatic chlamydial infection [25] and dissemination of C.trachomatis from the genital site to other intra-host sites has been documented in a murine model [26]. Moreover, both partners exhibited definite clinical symptoms of unilateral chronic bacterial conjunctivitis that likely were clinical manifestations of adult chlamydial conjunctivitis.

Surprisingly, the nvCT was identified in $100 \%$ of clinical specimens from either genital or extra-genital sites of both partners solely or as co-infection with wtCT in single sites (Tables 2, 3). These data provided a strong indirect evidence of possible association between the nvCT- infection and sub-fertility in this couple who were free from other STIs that are considered as potential bacterial species causing infertility including $N$. gonorrhoeae, U. urealyticum, and M. genitalium [27], as documented by conventional and real-time multiplex PCRs (data not shown). The screening for the relevant specific antibodies to these pathogens in sera of these patients also produced negative results.

In contrast to observations indicating the absence of significant differences in clinical manifestation between the nvCT and the wtCT strains $[14,15]$, we found no chlamydial genital symptoms in the male patient and only mild cervicitis in his female partner. Also contrary to the results of a high-risk city population attending a STIs clinic [15], the two individuals involved in the current study declared themselves perfectly healthy; the female patient did not have lower abdominal pain, pronounced vaginal discharge, frequent urination, post-coital bleeding, inter-menstrual bleeding, or any other complaints that were suggestive of a possible infection with Chlamydia. A complete absence of any symptoms of chlamydial genital infection in this couple caused by nvCT was distinct from the symptomatic infections observed for the wtCT strains by Bjartling et al. [15].

In fact, no major genetic difference was found between genomes of the nvCT and the wtCT strains suggesting that there is no alteration in biological fitness between two variants, which demonstrated high similarity with respect to epidemiological distribution and minimal differences in clinical signs in the vast majority of Chlamydia patients [28]. The transmission studies of the new variant of $C$ trachomatis in Sweden conducted for the past ten years also showed that nvCT is clonal and genetically stable. Moreover, difficulties in detecting the nvCT variant did not affect the complication rates [29].

Retrospective analysis of the records of 2012 obtained from the IVF Clinic, such as hormonal status for the female patient, and semen analyses of the male partner [4, 18] excluded any endocrine disorder in the woman, but the results of semen analysis of her male partner were normal only initially in 2012. The same analysis conducted two years later registered a dramatic decrease in at least two main parameters, sperm motility and spermatozoa vitality, that had markedly deteriorated by about 1.5 -fold compared with the initial test value, and were considered to be sub-normal as specified by the WHO [18]. Also a slight increase in ejaculate $\mathrm{pH}$ towards alkaline value was detected by this analysis. Although other sperm characteristics were in the normal range [18], the detected abnormalities in sperm could contribute to the failed results of the IVF procedure. These findings generally correlated with recent reports on the capability of human strains of wtCT to cause a significant decline in a number of vital and motile sperm in humans and mice, resulting in a marked decrease of its reproductive performance [5 - 8]. The development of these pathological changes found in the male patient took about 17 months.

These data indicated that nvCT may have a certain ability to infect both genital and extra-genital sites. Thus, including extra-genital sites in chlamydial diagnostic protocols can assist the efficient detection of this infection. The optimal detection of C.trachomatis in clinical specimens is possible only when appropriate targets for diagnostic assays are chosen [2]. A striking discrepancy in the ability of PCRs based on different targets used to detect C.trachomatis DNA in genital and extra-genital clinical specimens of our patients was observed (Tables 2, 3).

The important finding of our work was evidence of co-infection of the nvCT of genovar E and typical strains of wtCT of either E or D genovars in both patients during the second year of observation. The sequence analysis revealed the co-infection in most samples from conjunctiva and oropharynx (Table 3). We did not identify any previous 
publications reporting co-infection with nvCT and wtCT variants, although our data suggest that such combination may occur more often than expected. The role of a sole nvCT or a mixed nvCT and wtCT infection in human infertility requires further study. Recently, a simultaneous association of two wtCT genovars (either D and E or F and K) was reported with regard to the evaluation of treatment efficacy, reinfection and drug resistance [30 - 32]. In our case, multiple sexual contacts of the male patient apart from his marriage partner are a likely explanation for the finding of different genovars of C.trachomatis in the couple's specimens. Additionally, genovar E of the nvCT was first identified in this couple as a sole cause of Chlamydia infection, followed by two genovars of wtCT, D and E, identified during reexamination one year later in both partners (Table 3). This reinfection from a promiscuous male partner nullified the effect of the antibiotic treatment, and may have increased the likelihood of failure of the IVF. This case is a good example of recorded history of sexual behavior and clear transmission of C.trachomatis strains of different genovars among sex partners [33]. The use of SNP analysis within the ompA gene allowed further identification of the strains infecting both partners. The SNP at position 997 in the blood sample of the male patient was detected first during the screening of urogenital samples for C.trachomatis in a Swedish clinic in 1999-2000 [34]. We also identified the unique SNP at position 971 in genovar D in the specimens of both patients, indicating a similar origin of these strains. The presence of other polymorphisms, such as unique SNPs at positions 991 and 1020 in serovar D, in the samples of each individual patient suggested even wider co-infection that took place in this clinical case.

\section{CONCLUSION}

Finally, it can be concluded that the nvCT strain could be a cause of infertility in the male partner. Moreover, the deterioration in his semen quality appeared to begin when nvCT was the only strain detected, suggesting that it was a significant contributor. This illustrates the importance of including detection methods for nvCT strains in the investigation of infertility cases.

\section{ETHICS APPROVAL AND CONSENT TO PARTICIPATE}

The research protocol was approved by the Human Bioethics Committee of the Saratov Scientific and Research Veterinary Institute No. IRB00008288 (http://ohrp.cit.nih.gov/search/IrbDtl.aspx).

\section{HUMAN AND ANIMAL RIGHTS}

Animals did not participate in this research. All human research procedures followed were in accordance with the ethical standards of the committee responsible for human experimentation (institutional and national), and with the Helsinki Declaration of 1975, as revised in 2008.

\section{CONSENT FOR PUBLICATION}

Both participants provided written informed consent.

\section{CONFLICT OF INTEREST}

The authors declare no conflict of interest, financial or otherwise.

\section{ACKNOWLEDGEMENTS}

We are very grateful to Prof. Karl A. Western (NIAID/NIH) for his constant encouragement during the progress of this study. We thank Dr Nail Sh. Bogoutdinov for his excellent technical assistance. This work was supported by the Russian Science Foundation, Project No. 17-16-01099 (for Prof. V.A. Feodorova) and the Division of Intramural Research, National Institute of Allergy and Infectious Diseases, Baltimore, MD, 21205, USA (for Prof. T.C. Quinn).

\section{SUPPLEMENTARY MATERIAL}

Supplementary material is available on the publishers Website along with the published article.

\section{REFERENCES}

[1] Paavonen J, Eggert-Kruse W. Chlamydia trachomatis: Impact on human reproduction. Hum Reprod Update 1999; 5(5): $433-47$. [http://dx.doi.org/10.1093/humupd/5.5.433] [PMID: 10582782]

[2] Eley A. How to detect Chlamydia trachomatis in males? J Androl 2011; 32(1): 15-22. [http://dx.doi.org/10.2164/jandrol.110.010363] [PMID: 20798390] 
[3] Lal JA, Malogajski J, Verweij SP, et al. Chlamydia trachomatis infections and subfertility: Opportunities to translate host pathogen genomic data into public health. Public Health Genomics 2013; 16(1-2): 50-61. [http://dx.doi.org/10.1159/000346207] [PMID: 23548718]

[4] Fertility. Assessment and treatment for people with fertility problems NICE Clinial Guideline. $2^{\text {nd }}$ ed. 27 Sussex Place, Regent's Park, London NW1 4RG: The Royal College of Obstetricians and Gynaecologists 2013; pp. 1-555.

[5] Sellami H, Gdoura R, Mabrouk I, et al. A proposed mouse model to study male infertility provoked by genital serovar E, Chlamydia trachomatis. J Androl 2011; 32(1): 86-94. [http://dx.doi.org/10.2164/jandrol.109.009183] [PMID: 20539041]

[6] Carmichael JR, Tifrea D, Pal S, de la Maza LM. Differences in infectivity and induction of infertility: A comparative study of Chlamydia trachomatis strains in the murine model. Microbes Infect 2013; 15(3): 219-29.

[http://dx.doi.org/10.1016/j.micinf.2012.12.001] [PMID: 23287699]

[7] Eley A, Pacey AA, Galdiero M, Galdiero M, Galdiero F. Can Chlamydia trachomatis directly damage your sperm? Lancet Infect Dis 2005; 5(1): 53-7.

[http://dx.doi.org/10.1016/S1473-3099(04)01254-X] [PMID: 15620561]

[8] Hosseinzadeh S, Brewis IA, Eley A, Pacey AA. Co-incubation of human spermatozoa with Chlamydia trachomatis serovar E causes premature sperm death. Hum Reprod 2001; 16(2): 293-9. [http://dx.doi.org/10.1093/humrep/16.2.293] [PMID: 11157823]

[9] Pacey AA, Eley A. Chlamydia trachomatis and male fertility. Hum Fertil (Camb) 2004; 7(4): 271-6. [http://dx.doi.org/10.1080/14647270400016373] [PMID: 15621892]

[10] Ripa T, Nilsson P. A variant of Chlamydia trachomatis with deletion in cryptic plasmid: implications for use of PCR diagnostic tests. Euro Surveill 2006; 11(11): E061109.2. [PMID: 17213548]

[11] Ripa T, Nilsson PA. A Chlamydia trachomatis strain with a 377-bp deletion in the cryptic plasmid causing false-negative nucleic acid amplification tests. Sex Transm Dis 2007; 34(5): 255-6. [PMID: 17483723]

[12] Unemo M, Clarke IN. The Swedish new variant of Chlamydia trachomatis. Curr Opin Infect Dis 2011; 24(1): 62-9. [http://dx.doi.org/10.1097/QCO.0b013e32834204d5] [PMID: 21157332]

[13] Harris SR, Clarke IN, Seth-Smith HM, et al. Whole-genome analysis of diverse Chlamydia trachomatis strains identifies phylogenetic relationships masked by current clinical typing. Nat Genet 2012; 44(4): 413-419, S1. [http://dx.doi.org/10.1038/ng.2214] [PMID: 22406642]

[14] Marions L, Rotzen-Ostlund M, Grillner L, et al. High occurrence of a new variant of Chlamydia trachomatis escaping diagnostic tests among STI clinic patients in Stockholm, Sweden. Sex Transm Dis 2008; 35(1): 61-4. [http://dx.doi.org/10.1097/OLQ.0b013e318141feac] [PMID: 17762244]

[15] Bjartling C, Osser S, Johnsson A, Persson K. Clinical manifestations and epidemiology of the new genetic variant of Chlamydia trachomatis. Sex Transm Dis 2009; 36(9): 529-35.

[http://dx.doi.org/10.1097/OLQ.0b013e3181a8cef1] [PMID: 19617866]

[16] Workowski KA, Berman S. CDC Sexually Transmitted Diseases Treatment Guidelines 2010. Available from: http://www.cdc.gov/mmwr/preview/mmwrhtml/rr5912a1.htm

[17] Lanjouw E, Ossewaarde JM, Stary A, Boag F, van der Meijden WI. 2010 European guideline for the management of Chlamydia trachomatis infections. Int J STD AIDS 2010; 21(11): 729-37. [http://dx.doi.org/10.1258/ijsa.2010.010302] [PMID: 21187352]

[18] WHO Laboratory manual for the examination and processing of human semen. The $5^{\text {th }}$ ed. 20 Avenue Appia, 1211 Geneva 27 , Switzerland: World Health Organization 2010; pp. 1-270.

[19] Kutlin AV, Shatkin AA, Drobyshevskaia EI. Immunodiagnostic preparations based on monoclonal antibodies to Chlamydia trachomatis.. Zh Mikrobiol Epidemiol Immunobiol 1996; 6(6): 42-4.

[PMID: 9103077]

[20] Shipitsyna E, Zolotoverkhaya E, Agné-Stadling I, et al. First evaluation of six nucleic acid amplification tests widely used in the diagnosis of Chlamydia trachomatis in Russia. J Eur Acad Dermatol Venereol 2009; 23(3): 268-76.

[http://dx.doi.org/10.1111/j.1468-3083.2008.03038.x] [PMID: 19207643]

[21] Catsburg A, van Dommelen L, Smelov V, et al. TaqMan assay for Swedish Chlamydia trachomatis variant. Emerg Infect Dis 2007; 13(9): 1432-4. [http://dx.doi.org/10.3201/eid1309.070263] [PMID: 18252135]

[22] Quint KD, van Doorn LJ, Kleter B, et al. A highly sensitive, multiplex broad-spectrum PCR-DNA-enzyme immunoassay and reverse hybridization assay for rapid detection and identification of Chlamydia trachomatis serovars. J Mol Diagn 2007; 9(5): 631-8. [http://dx.doi.org/10.2353/jmoldx.2007.070011] [PMID: 17872971]

[23] Dean D, Oudens E, Bolan G, Padian N, Schachter J. Major outer membrane protein variants of Chlamydia trachomatis are associated with severe upper genital tract infections and histopathology in San Francisco. J Infect Dis 1995; 172(4): 1013-22. 
[http://dx.doi.org/10.1093/infdis/172.4.1013] [PMID: 7561174]

[24] Recommendations for the laboratory-based detection of Chlamydiatrachomatis and Neisseria gonorrhoeae-2014 CDC MMWR Recomm Rep 2014 Mar 14; 63(RR02) Available at: http://www.cdc.gov/mmwr/preview/mmwrhtml/rr6302a1.htm

[25] Pashko IuP, Zigangirova NA, Petiaev IuM, et al. Modern aspects of diagnostics of chronic chlamydiosis caused by persisting forms of Chlamydia. Zh Mikrobiol Epidemiol Immunobiol 2009; 4(4): 89-93. [Russian.]. [PMID: 19718830]

[26] Whittum-Hudson JA, Gerard HC, Clayburne G, et al. A non-invasive murine model of Chlamydia induced reactive arthritis. Discussion 1999; $66(50 \mathrm{~S}): 5 \mathrm{~S}-56 \mathrm{~S}$.

[27] Abusarah EA, Awwad ZM, Charvalos E, Shehabi AA. Molecular detection of potential sexually transmitted pathogens in semen and urine specimens of infertile and fertile males. Diagn Microbiol Infect Dis 2013; 77(4): 283-6. [http://dx.doi.org/10.1016/j.diagmicrobio.2013.05.018] [PMID: 24079950]

[28] Unemo M, Seth-Smith HMB, Cutcliffe LT, et al. The Swedish new variant of Chlamydia trachomatis: Genome sequence, morphology, cell tropism and phenotypic characterization. Microbiology 2010; 156(Pt 5): 1394-404. [http://dx.doi.org/10.1099/mic.0.036830-0] [PMID: 20093289]

[29] Dahlberg J, Hadad R, Elfving K, et al. Ten years transmission of the new variant of Chlamydia trachomatis in Sweden: Prevalence of infections and associated complications. Sex Transm Infect 2018; 94(2): 100-4. [http://dx.doi.org/10.1136/sextrans-2016-052992] [PMID: 28724744]

[30] Molano M, Meijer CJ, Morré SA, Pol R, van den Brule AJ. Combination of PCR targeting the VD2 of omp1 and reverse line blot analysis for typing of urogenital Chlamydia trachomatis serovars in cervical scrape specimens. J Clin Microbiol 2004; 42(7): 2935-9. [http://dx.doi.org/10.1128/JCM.42.7.2935-2939.2004] [PMID: 15243041]

[31] Lima HE, Oliveira MB, Valente BG, et al. Genotyping of Chlamydia trachomatis from endocervical specimens in Brazil. Sex Transm Dis 2007; 34(9): 709-17. [http://dx.doi.org/10.1097/01.olq.0000258399.27873.d9] [PMID: 17414937]

[32] Gharsallah H, Frikha-Gargouri O, Sellami H, Besbes F, Znazen A, Hammami A, et al. Chlamydia trachomatis genovar distribution in clinical urogenital specimens from Tunisian patients: High prevalence of C. trachomatis genovar E and mixed infections. BMC Infect Dis 2012; 12: 333. [http://dx.doi.org/10.1186/1471-2334-12-333] [PMID: 23198910]

[33] Batteiger BE, Wan R, Williams JA, et al. Novel Chlamydia trachomatis strains in heterosexual sex partners, Indianapolis, Indiana, USA. Emerg Infect Dis 2014; 20(11): 1841-7. [http://dx.doi.org/10.3201/2011.140604] [PMID: 25340463]

[34] Jurstrand M, Falk L, Fredlund H, et al. Characterization of Chlamydia trachomatis ompl genotypes among sexually transmitted disease patients in Sweden. J Clin Microbiol 2001; 39(11): 3915-9. [http://dx.doi.org/10.1128/JCM.39.11.3915-3919.2001] [PMID: 11682507]

(C) 2018 Feodorova et al.

This is an open access article distributed under the terms of the Creative Commons Attribution 4.0 International Public License (CC-BY 4.0), a copy of which is available at: (https://creativecommons.org/licenses/by/4.0/legalcode). This license permits unrestricted use, distribution, and reproduction in any medium, provided the original author and source are credited. 